\title{
On the ionization loss spectra of high-energy channeled negatively charged particles
}

\author{
S. V. Trofymenko ${ }^{1,2, a}$, I. V. Kyryllin ${ }^{1,2, b}$ \\ ${ }^{1}$ Akhiezer Institute for Theoretical Physics, National Science Center "Kharkov Institute of Physics and Technology", Akademicheskaya Str., 1, \\ Kharkiv 61108, Ukraine \\ ${ }^{2}$ V.N. Karazin Kharkiv National University, Svobody Sq. 4, Kharkiv 61022, Ukraine
}

Received: 30 April 2020 / Accepted: 5 June 2020 / Published online: 31 July 2020

(C) The Author(s) 2020

\begin{abstract}
The ionization loss spectra of high-energy negatively charged particles which move in the planar channeling mode in a silicon crystal are studied with the use of numerical simulation. The case when the crystal thickness is on the order of the dechanneling length $l_{d}$ is considered. It is shown that in this case the shape of the spectrum noticeably depends on $l_{d}$. The evolution of various characteristic parameters of the spectrum with the change of $l_{d}$ is investigated. A method of the experimental determination of $l_{d}$ on the basis of the measurement of the ionization loss spectrum is proposed.
\end{abstract}

\section{Introduction}

When a charged particle penetrates through matter it loses part of its energy on excitation and ionization of the atoms, which makes up the particle ionization loss. In sufficiently thin targets the value of such a loss is stochastic. It is distributed according to the law, which was first derived in [1] and further elaborated in [2-4]. In amorphous targets such a distribution (or spectrum), known as the Landau distribution, has a single maximum, which corresponds to the most probable value of the particle energy loss (MPEL).

If a charged particle enters a crystalline target at a small angle with respect to crystallographic axes or planes, the channeling effect may occur [5]. It happens as a result of the particle capture by the potential wells formed by the atomic strings or planes. The channeling motion of a particle in a crystal can break down due to its incoherent scattering either on thermal vibrations of the atoms (mostly for negative particles) or on the subsystem of the atomic electrons (mostly for positive particles). In the case of negatively charged particles the decrease in the number of channeled particles with

\footnotetext{
a e-mail: trofymenko@kipt.kharkov.ua (corresponding author)

b e-mail: kirillin@kipt.kharkov.ua
}

the crystal thickness behaves almost exponentially [6]. The distance on which the number of channeled particles in the incident beam decreases by a factor of $e$ (due to the particle transition to the over-barrier regime of motion) is known as the dechanneling length $l_{d}$.

For positive particles (protons, positrons etc.) the channeling effect results in the decrease of the particle MPEL compared to the case when the crystal is disoriented (in this case the crystal resembles an amorphous medium) and the particle penetrates the whole target in the over-barrier regime. It is associated with the fact that, due to the repulsive nature of the force between the particle and the atomic stings or planes, the probability for the particle to experience close collisions with the atoms decreases. Such an effect was observed in [710]. In $[8,9]$ the measured ionization loss spectra for negative particles ( $\pi^{-}$mesons) were presented as well. However, here the condition $l_{d} \ll L$ (where $L$ is the thickness of the crystalline target) took place. This led either to the absence of any noticeable channeling impact upon the MPEL value [8] or to a very small effect of this kind [9].

In the present work, on the basis of the numerical simulation, we study the ionization loss spectra of negatively charged particles under condition when the value of $l_{d}$ is on the order of $L$. It is shown that in this case the shapes of the spectra noticeably differ from the one of the conventional Landau distribution and are rather sensitive to the ratio $l_{d} / L$. The possibility of the experimental determination of $l_{d}$ from the measurement of various characteristics of the ionization loss spectra is studied.

Generally, channeling of negatively charged particles can be a useful tool for beam manipulation, radiation production in crystals or crystalline undulators and for positron production. The length of dechanneling is the parameter which allows one to determine the thickness of the crystal up to which the particles move inside it in the channeling mode. Attempts to determine the dechanneling length of negatively 
Table 1 Values of the coefficient of proportionality between the dechanneling length and the energy of the charged particles

\begin{tabular}{lllll}
\hline Plane & Particles & $E[\mathrm{GeV}]$ & $\xi[\mu \mathrm{m} / \mathrm{GeV}]$ & References \\
\hline$(110)$ & $e^{-}$ & & 17.8 & {$[11]$} \\
$(110)$ & $e^{-}$ & 0.855 & 21.1 & {$[12]$} \\
$(110)$ & $e^{-}$ & 0.855 & 48.0 & {$[13]$} \\
$(110)$ & $e^{-}$ & 0.855 & 9.7 & {$[14]$} \\
$(110)$ & $e^{-}$ & 1.2 & 24.2 & {$[15]$} \\
$(110)$ & $\pi^{-}$ & 150 & 6.2 & {$[16]$} \\
$(111)$ & $e^{-}$ & & 23.6 & {$[11]$} \\
$(111)$ & $e^{-}$ & 0.855 & 23.7 & {$[17]$} \\
$(111)$ & $e^{-}$ & 0.855 & 15.9 & {$[14]$} \\
$(111)$ & $e^{-}$ & $3.35-14$ & 15.3 & {$[18]$} \\
$(111)$ & $e^{-}$ & $0.5-100$ & 27 & {$[19]$} \\
$(111)$ & $e^{-}$ & 50 & 6.6 & {$[20]$} \\
\hline
\end{tabular}

charged particles have been repeatedly made previously by means of theoretical calculations, numerical simulations and experiments. In [11], on the basis of the comparison of the root mean square angle of multiple scattering with the critical angle of planar channeling, it was found that the dechanneling length is linearly proportional to the energy of the charged particles: $l_{d}=\xi E$. Further estimates and measurements were aimed at determining the coefficient of proportionality $\xi$

In Table 1 we present the estimates for $\xi$ in a Si crystal that we found in the previous works. One may see that the value of $\xi$ varies in a rather wide range and still requires clarification.

There are several experimental methods which allow determining the value of the dechanneling length in a crystal. These methods are associated with the detection of the yield of the atomic electrons knocked out from the crystals [15], with the measurement of the low-energy channeling radiation or high-energy bremsstrahlung [12] and with the measurement of the deflection angle of the particles after their passage through a bent crystal [16-18]. In the present paper we propose a new method of the dechanneling length determination through the analysis of the particle ionization energy loss spectra in crystals.

\section{Outline of the simulation method}

As a result of simulation of the particle motion in the crystal we find the particle trajectory in the field of periodically located atomic planes. For definiteness, simulation was performed for the motion of $150 \mathrm{GeV} / c \pi^{-}$mesons in (110) planar channels of a silicon crystal. A secondary beam of $\pi^{-}$mesons with such an energy can be obtained at the SPS
CERN accelerator. The simulation code is analogous to the one applied in Refs. [21-25]. However, presently it is the potential of the crystalline atomic planes which plays the role of the external field, while in [21-25] it was the potential of the atomic strings. Thus, not a two-dimensional, but a one-dimensional equation of motion is solved. The code computes the trajectory of a high-energy charged particle in the potential of the continuous atomic planes in the DoyleTurner approximation through the numerical integration of the equation of motion. It takes into account the incoherent scattering caused by thermal vibrations of the atoms and scattering on the electrons. Other kinds of incoherent scattering were not taken into account considering the small crystal thickness.

For simulation of the particle ionization loss value on each infinitesimal interval of its trajectory we apply the expression for the cross-section $d \sigma / d E$ of the particle energy loss upon inelastic scattering on a silicon atom, derived in [26]. It is a model cross-section, which includes both the terms describing the resonant excitations of the atomic shells $(\mathrm{K}, \mathrm{L}$ and $\mathrm{M})$ and the terms associated with the particle non-resonant Coulomb scatterings on the atomic electrons. In [26,27] such a cross-section was applied for calculation of the ionization loss spectra in disoriented silicon targets and a rather nice coincidence with the experimental data was achieved.

The probability of a certain particle energy loss value $E$ in a collision is $d w=\sigma^{-1}(d \sigma / d E) d E$, where $\sigma$ is the total collision cross-section. It is applied for simulation of the particle energy loss on the interval $d x$ of its trajectory in the crystal in case the particle experiences a collision on this interval. The probability of such a collision $d P=n_{\mathrm{eff}} \sigma d x$ is defined by the effective atomic electron density $n_{\text {eff }}$ on the interval $d x$. Note that $n_{\text {eff }}$ is different form the local atomic electron density $n(\mathbf{r})$ at the particle position $\mathbf{r}$ as well as from the mean electron density $n$ of the crystal. We will presently apply the relation [5]:

$n_{\mathrm{eff}}=(1-\alpha) n+\alpha n(\mathbf{r})$

and choose $\alpha=1 / 2$. Such a choice accounts for the approximate equipartition between the contributions of the close and distant collisions to the particle ionization loss at high energies $[5,28,29] .{ }^{1}$

Figure 1 shows a comparison of the simulation results for the ionization loss spectrum of $150 \mathrm{GeV} / \mathrm{c} \pi^{-}$mesons in a disoriented silicon crystal of $L=1 \mathrm{~mm}$ thickness (which is analogous to an amorphous target) with the corresponding calculation on the basis of the analytical theory elaborated in [3]. This theory demonstrates a nice coincidence with the available experimental data, e. g., [26]. The function $f_{d}(E)$ on the vertical axis of the figure is a probability distribution

\footnotetext{
${ }^{1}$ For the accurate treatment of the mean value of the channeled particle ionization loss, without the equipartition assumption, see [30].
} 


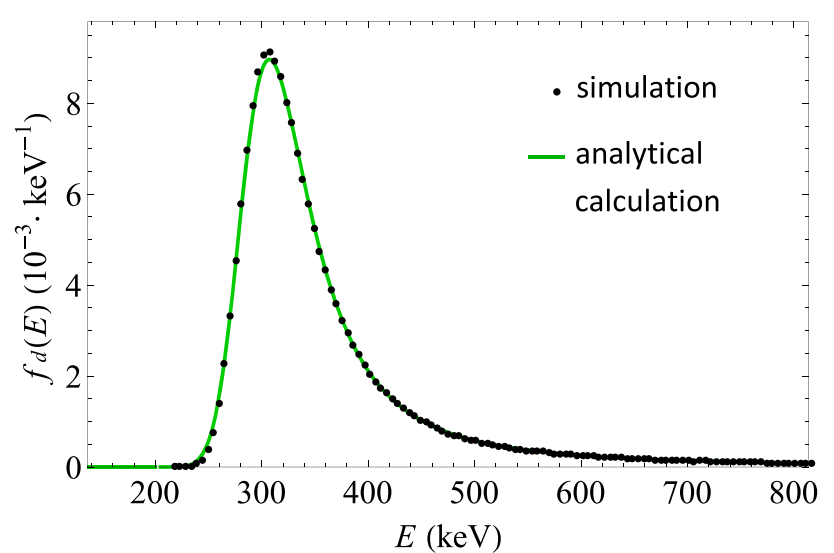

Fig. 1 Ionization loss spectrum of $150 \mathrm{GeV} / \mathrm{c} \pi^{-}$mesons in a disoriented $1 \mathrm{~mm}$ silicon target

of the particle ionizaiton loss value $E$ in the target, which is normalized to unity. The figure indicates a very nice workability of our simulation method at least in the particular case of a disoriented crystal $\left(n_{\text {eff }}=n\right)$. We have also compared the results provided by our code with the results of measurements of the ionization loss spectra of $15 \mathrm{GeV}$ protons in oriented silicon crystals [9]. The comparison showed a good qualitative agreement between the simulation and the experimental results. Some quantitative discrepancies can be attributed to a relatively large beam divergence and some uncertainty in the crystal alignment with respect to the beam direction, which took place in the experiment. Nevertheless, further experimental study with the use of a precisely aligned beam having a divergence within the critical angle of planar channeling (available, e. g., at SPS) is encouraged for a deeper test of the present simulation method. Particularly, this concerns clarification of the precise value of $\alpha$ in (1).

\section{Ionization loss spectra of channeled negatively charged particles}

In this section we present the results of simulation of the ionization loss spectra for $150 \mathrm{GeV} / \mathrm{c} \pi^{-}$mesons in a thin oriented silicon crystal and study the dependence of the properties of these spectra on the dechanneling length of the particles. Special attention is drawn to investigation of the possibility to apply these spectra for the measurement of the dechanneling length.

In a real experiment it is the particle energy $\varepsilon$ and the crystal thickness $L$ that can be arbitrarily chosen from the technically available range, while the dechanneling length is fixed by the choice of $\varepsilon$. In the present work, however, we consider $l_{d}$ as a free parameter and perform the simulation for the fixed values of $\varepsilon$ and $L$. Such an approach is reasonable in terms of its application for the determination of $l_{d}$ on

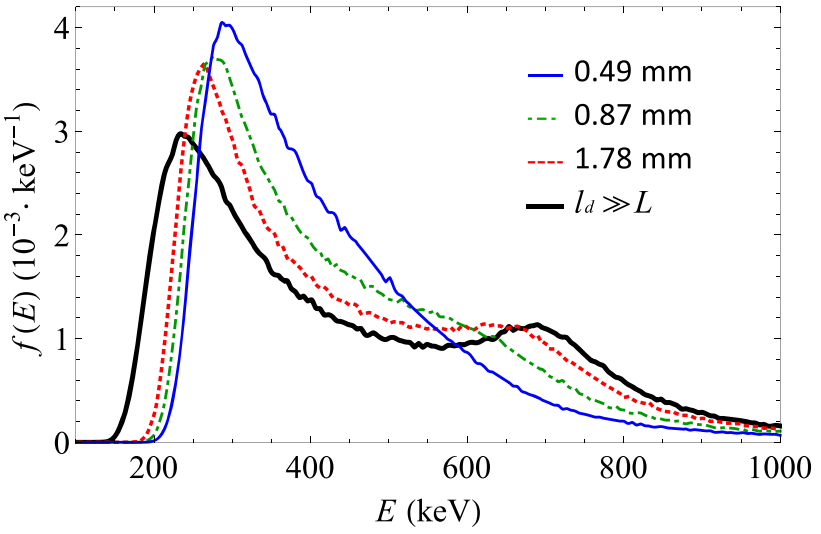

Fig. 2 Ionization loss spectra of $150 \mathrm{GeV} / \mathrm{c} \pi^{-}$mesons in $1 \mathrm{~mm}$ silicon target for different values of $l_{d}$ (specified in the legend). The particles incident along (110) plane

the basis of the measured ionization loss spectra. Namely, suppose that a simulation of a set of spectra corresponding to different values of $l_{d}$ is performed (for the fixed $\varepsilon$ and $L$ ) and a comparison with the experimentally measured spectrum is made. In principle, it is possible to deduce the value of $l_{d}$ as the one corresponding to the spectrum from the simulated set, which demonstrates the best coincidence with the experimental spectrum. Such an approach is applicable if the parameters describing the spectra noticeably vary with the change of $l_{d}$. We show that it is the case if the crystal thickness $L$ is on the same order of magnitude as $l_{d}$. On the basis of Table 1 for the considered value of the particle energy we choose $L=1 \mathrm{~mm}$.

At the present stage our major aim is to study the principal workability of such an approach and we will simplify the initial conditions where possible. Particularly, the divergence of the $\pi^{-}$meson beam will be neglected and all the particles will be assumed to enter the crystal parallel to a certain set of crystalline planes. As an example, a set of (110) planes is presently chosen. From the point of view of the experimental design, the condition of a small beam divergence can be fulfilled by the preliminary collimation of the beam in a certain direction. The dechanneling length is varied in a most natural way by changing the intensity of the particle incoherent multiple scattering on atoms. We sequentially performed simulations for different values of the coefficient of proportionality between the mean square of the incoherent multiple scattering angle and the path length travelled by the particle in the crystal. The larger this coefficient, the faster the particle exits the channeling mode. In the simulations we determined the dechanneling length as the crystal thickness at which $1-\frac{1}{e}$ of the particles leaves the planar channel. In our case the length of dechanneling is defined without the account of the process of rechanneling. The latter process is, certainly, taken into account in the modelling of the particle motion in the crystal. 
Figure 2 shows the simulation results for the ionization loss spectra of $150 \mathrm{GeV} / \mathrm{c} \pi^{-}$mesons incident along (110) plane on a silicon crystal. Different spectra correspond to different values of the dechanneling length. The simulation was performed for the values of $l_{d}$ from $0.49 \mathrm{~mm}<L$ (thin solid blue line) to $1.78 \mathrm{~mm}>L$ (dotted red line). The spectrum corresponding to the case when dechanneling can be neglected is presented as well (thick solid black line). The figure shows that in the considered case the ionization loss spectra significantly differ from the spectrum typical for a disoriented crystal (Fig. 1). We see that a noticeable change of the spectrum shape accompanies the change of $l_{d}$. This provides a principal possibility to apply the measurement of the ionization loss spectrum for determination of $l_{d}$.

Let us by the way note that, from the other hand, Fig. 2 indicates that under discussed conditions $\left(L \sim l_{d}\right)$ the ionization loss spectra of high-energy channeled particles vary with the particle energy (for a fixed $L$ ). Indeed, due to the proportionality of $l_{d}$ to $\varepsilon$ the change of $l_{d}$ can be accomplished by the change of $\varepsilon$. This is a somewhat new effect, which is not typical neither for amorphous targets (or disoriented crystals) or oriented crystals in the case $L \ll l_{d}$. In the latter cases, due to the density effect [31], the ionization loss spectrum of high-energy particles is independent on the particle energy (at least for the values of $E$ not much larger than MPEL).

It is also worth paying special attention to the spectrum at $L \ll l_{d}$ when the majority of the incident particles penetrate the whole crystal in the channeling mode. According to simulation, this spectrum has a two-humped structure. A more detailed study, involving separate simulations of the spectra for incident particles with the different impact parameters, provides the following explanation for it. The intuitively expected right maximum at about $690 \mathrm{keV}$ is produced by the particles with sufficiently small impact parameters with respect to the plane. Most of the time such particles move in the region of high atomic electron density. The second maximum, which is shifted to the left with respect to the MPEL value for the disoriented crystal, is, probably, somewhat counterintuitive (since for negatively charged particles just the effect of the ionization loss increase might be expected). Nevertheless, it can be explained by the particles with relatively large impact parameters which enter the crystal in the vicinity of the center of the interplanar gaps. The point is that in the course of their oscillatory motion such particles, being attracted by the planes, pass through them having the maximal speed. In the gaps between the planes, on the contrary, they are decelerated. Hence, such particles spend most of the time in the gaps, where the electron density is rather low, and the channeling effect results in the decrease of their ionization loss.

Let us also note that in general it is not possible to fit the spectrum at $L \ll l_{d}$ by only two Landau distributions with the values of MPEL corresponding to the maxima of the spectrum. It is rather a superposition of a continuous series of such distributions with the values of MPEL in the interval between these maxima, associated with the incident particles having various impact parameters. At $L \sim l_{d}$ the spectra have even more complicated structure. Presently in the first part of the crystal the particles still loose their energy moving in the channeling mode. But in the rest of the crystal some particles become overbarrier and their ionization loss occurs like in disoriented crystals (or amorphous targets). Therefore at $L \sim$ $l_{d}$ the spectrum is a convolution of the spectra typical for channeled and overbarrier particles. Moreover, due to the fact that the dechanneling rate varies with the impact parameter of the incident particle, such a convolution should be separately performed for different values of this parameter. The resultant spectrum is the integral with respect to the impact parameters.

\section{Characteristic parameters of the spectra sensitive to $l_{d}$}

As noted, in the discussed method, in order to experimentally define the value of $l_{d}$ it is necessary to compare the measured spectrum with the simulation results. In this case the simulated spectra are supposed to be improved by taking into account such experimental parameters as the beam divergence, its possible misalignment etc. in order to achieve the best coincidence of the shape of the experimental spectrum with the one simulated for a certain value of $l_{d}$. The latter value is defined as the dechanneling length which takes place in the experiment. Instead of comparing the shapes of the spectra (which are not actually quantitative characteristics) it might be more convenient to compare various numerical parameters characterising the spectra, which are sufficiently sensitive to the value of $l_{d}$. In the present section we discuss a series of such parameters.

For instance, the role of such a parameter can be played by the ratio $\eta_{L / R}=f\left(E_{L}\right) / f\left(E_{R 0}\right)$. Here $E=E_{L}$ is the position of the left maximum of the function $f(E)$ (for $l_{d}<$ $1.4 \mathrm{~mm}$ it is the only maximum) which slightly shifts with the change of $l_{d}$, see Fig. 2. By $f\left(E_{R 0}\right)$ we denote the value of $f(E)$ at the position of the right maximum at $l_{d} \gg L$, which is presently $E_{R 0} \approx 690 \mathrm{keV}$.

Figure 3 shows the dependence of $\eta_{L / R}$ on $l_{d}$. The simulated dependence is nicely fitted by the function $a+b / x^{c}$ with $a=2.87, b=1.78$ and $c=1.83$. We see that the parameter $\eta_{L / R}$ is rather sensitive to the dechanneling length value in the region $l_{d}<L$ and can be applied for determination of $l_{d}$, provided it belongs to this region. If $l_{d}$ exceeds the crystal thickness, the parameter $\eta_{R / L}$ saturates and becomes inconvenient for the measurement of $l_{d}$. For instance, if the experimentally measured value of $\eta_{L / R}$ is around 4 , it is hard to deduce $l_{d}$ from Fig. 3 without a considerable uncertainty. 


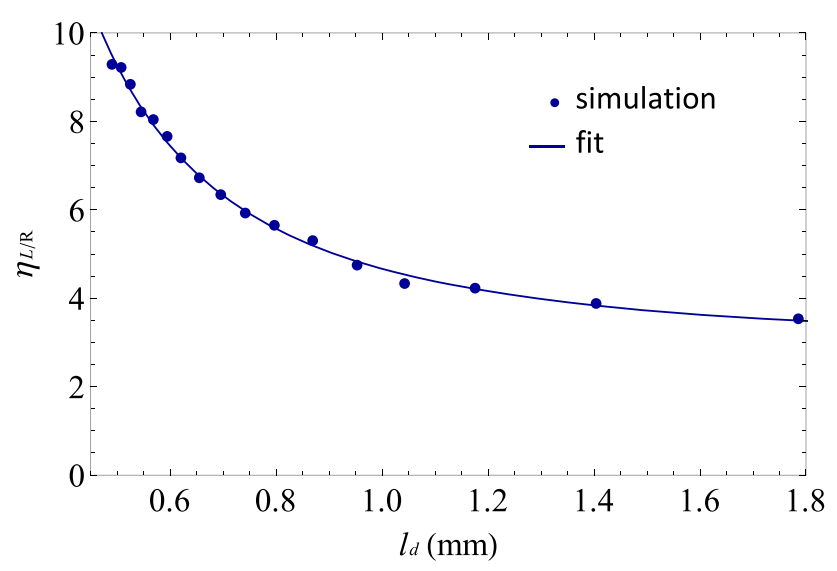

Fig. 3 Dependence of the ratio $f\left(E_{L}\right) / f\left(E_{R 0}\right)$ on the dechanneling length in $1 \mathrm{~mm}$ silicon crystal

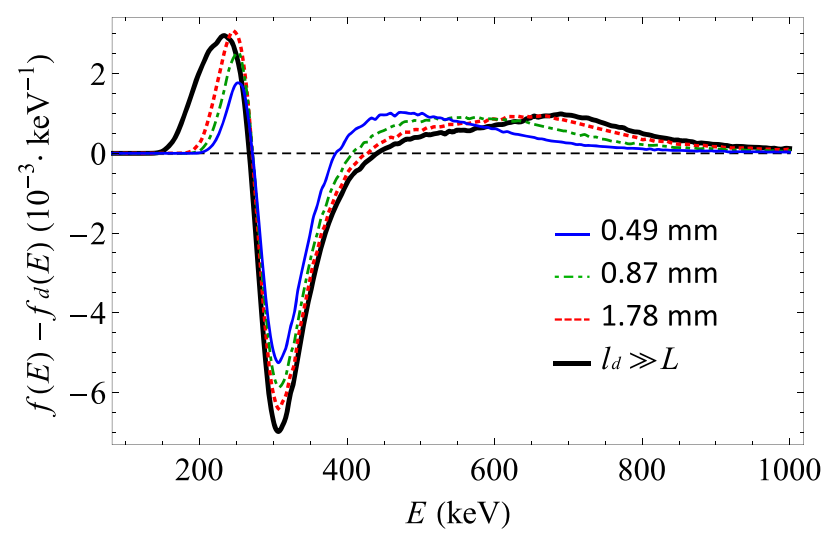

Fig. 4 Difference of the spectra presented in Figs. 2 and 1

In this case a thicker crystal can be applied, in which $\eta_{L / R}$ is still far from saturation.

The next possibility is to consider the function $f_{\text {dif }}(E)$ which is obtained by subtraction of the spectrum $f_{d}(E)$ in the disoriented crystal from the spectrum $f(E)$ in the oriented one. Figure 4 demonstrates the result of this subtraction for different values of $l_{d}$ (the same as in Fig. 2). The use of the function $f_{\text {dif }}(E)$ can be motivated by the possibility to eliminate the common background which can exist in the measured $f_{d}(E)$ and $f(E)$ spectra. In this case it is possible to use the value of the function $f_{\text {dif }}$ at the point of its minimum at $E=E_{m} \approx 300 \mathrm{keV}$ as a parameter sensitive to $l_{d}$. Figure 5 demonstrates the corresponding dependence. The fitting is performed with the use of the function $a+b\left(1-\exp \left[c x^{d}\right]\right)$ with $a=-2.78, b=-3.79, c=-1.92$ and $d=0.85$. In this case, however, a rather large measurement statistics may be needed (large number of the incident particles) since the change of $f_{\text {dif }}\left(E_{m}\right)$ is rather small and a sufficiently low statistical uncertainty in its measurement is required.

The parameters $\eta_{L / R}$ and $f_{\text {dif }}\left(E_{m}\right)$ demonstrate certain statistical fluctuations which originate from the same fluctuations of the measured or simulated values of $f(E)$. It

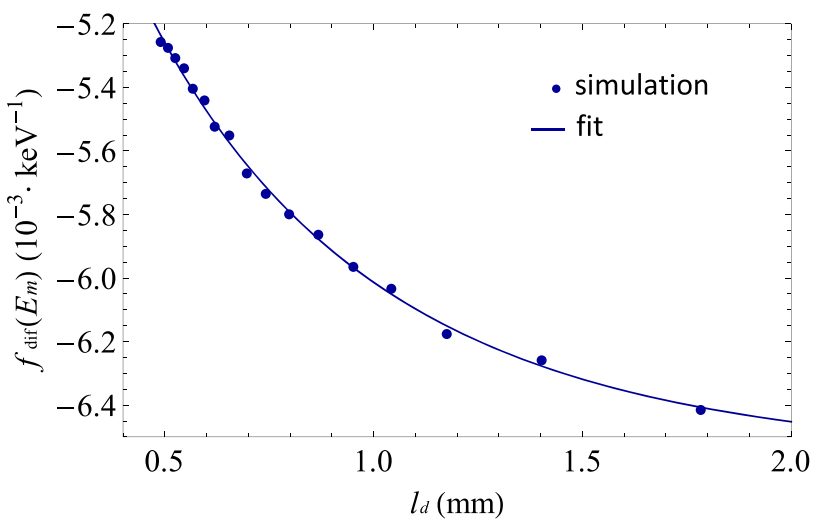

Fig. 5 Dependence of $f_{\text {dif }}\left(E_{m}\right)$ on $l_{d}$

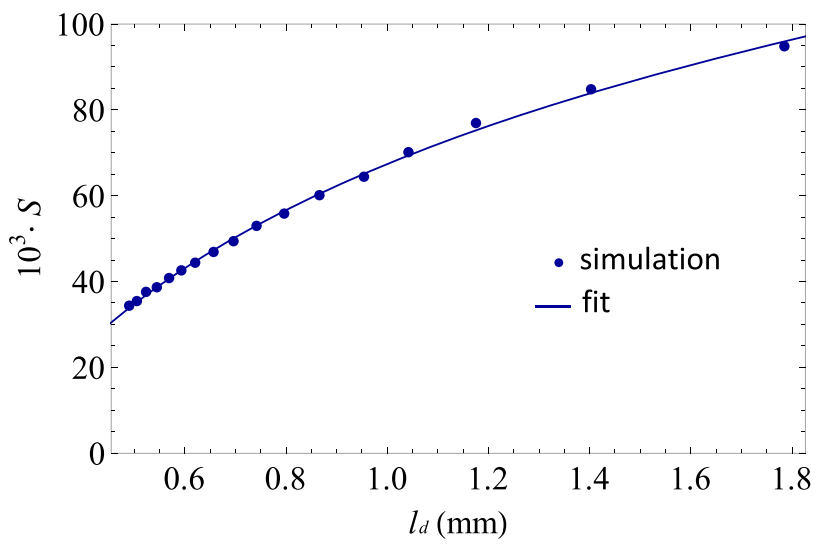

Fig. 6 Dependence of $S$ on $l_{d}$

is possible to introduce a parameter which is less prone to such fluctuations and, therefore, requires less statistics for its measurement. As an example of such a parameter the area $S$ under the curve $f(E)$ in a certain interval of $E$ can be chosen. Presently we will calculate the area which corresponds to the region $E<E_{L 0}$, where $E_{L 0} \approx 250 \mathrm{keV}$ defines the position of the left maximum of the spectrum at $l_{d} \gg L$. Figure 6 shows the dependence of $S$ on the dechanneling length in this case. The fitting on the figure is performed with the use of the function $a+b x^{c}$ with $a=-476.57$, $b=543.87$ and $c=0.089$. The similar dependence can be obtained if choose, e. g., the region $E_{R 0}<E<1.5 E_{R 0}$ with $E_{R 0} \approx 690 \mathrm{keV}$ being, as previously, the right maximum position of the same spectrum.

\section{Conclusions}

In the present work the properties of the ionization loss spectra generated by negatively charged particles channeled in silicon crystals were studied on the basis of numerical simulation. The crystals of thickness $L$ on the order of the dechan- 
neling length $l_{d}$ were considered. The applied method of the ionization loss simulation was based on the model crosssection for the energy transfer by an incident particle to atomic electrons, derived in [26]. It is shown that under considered condition $\left(L \sim l_{d}\right.$ ) the shape of the ionization loss spectrum is sensitive to the value of $l_{d}$ (and, hence, to the particle energy $\varepsilon$ as well). The dependence of the spectra on $l_{d}$ was investigated for the case of $150 \mathrm{GeV} / \mathrm{c} \pi^{-}$mesons in (110) planar channels of a $1 \mathrm{~mm}$ silicon crystal. A method of experimental determination of the dechanneling length, based on the measurement of the ionization loss spectrum, is proposed. This method is associated with the comparison of the experimentally measured spectrum with the series of spectra simulated for different values of $l_{d}$. It is shown that such a comparison can be made with the use of various numerical parameters, characterizing the spectrum, which noticeably depend on $l_{d}$.

Acknowledgements We recognize the support of the National Academy of Sciences of Ukraine (budget program "Support for the Development of Priority Areas of Scientific Research" (6541230), projects C-2/502020 and F30-2020).

Data Availability Statement This manuscript has no associated data or the data will not be deposited. [Authors' comment: The paper is theoretical and does not contain any data obtained in the result of experimental measurements.]

Open Access This article is licensed under a Creative Commons Attribution 4.0 International License, which permits use, sharing, adaptation, distribution and reproduction in any medium or format, as long as you give appropriate credit to the original author(s) and the source, provide a link to the Creative Commons licence, and indicate if changes were made. The images or other third party material in this article are included in the article's Creative Commons licence, unless indicated otherwise in a credit line to the material. If material is not included in the article's Creative Commons licence and your intended use is not permitted by statutory regulation or exceeds the permitted use, you will need to obtain permission directly from the copyright holder. To view a copy of this licence, visit http://creativecomm ons.org/licenses/by/4.0/.

Funded by SCOAP ${ }^{3}$.

\section{References}

1. L.D. Landau, J. Phys. USSR 8, 201 (1944)

2. P.V. Vavilov, Sov. Phys. JETP 5, 749 (1957) [P.V. Vavilov, Zh. Exp. Teor. Fiz. 32, 920 (1957)]
3. P. Shulek, B.M. Golovin, L.A. Kuliukina et al., Sov. J. Nucl. Phys. 4, 400 (1967) [P. Shulek, B.M. Golovin, L.A. Kuliukina et al., Yad. Fiz. 4, 564 (1967)]

4. H. Bichsel, Rev. Mod. Phys. 60, 663 (1988)

5. J. Lindhard, Danske Vid. Selsk. Mat. Fys. Medd. 34, 14 (1965)

6. V.V. Tikhomirov, Eur. Phys. J. C 77, 483 (2017)

7. O. Fich, J.A. Golovchenko, K.O. Nielsen et al., Phys. Rev. Lett. 36, 1245 (1976)

8. H. Esbensen, O. Fich, J.A. Golovchenko et al., Nucl. Phys. B 127, 281 (1977)

9. H. Esbensen, O. Fich, J.A. Golovchenko et al., Phys. Rev. B 18, 1039 (1978)

10. S. Pape Møller, V. Biryukov, S. Datz et al., Phys. Rev. A 64, 032902 (2001)

11. V. Baier, V. Katkov, V. Strakhovenko, Electromagnetic Processes at High Energies in Oriented Single Crystals (World Scientific, Singapore, 1998)

12. H. Backe, P. Kunz, W. Lauth, A. Rueda, Nucl. Instr. Meth. Phys. Res. B 266, 3835 (2008)

13. W. Lauth, H. Backe, P. Kunz, A. Rueda, Int. J. Mod. Phys. A 25, $136(2010)$

14. A. Kostyuk, A. Korol, A. Solov'yov, W. Greiner, J. Phys. B 44, $075208(2011)$

15. V.I. Vit'ko, G.D. Kovalenko, Sov. Phys. JETP 67, 2141 (1988)

16. W. Scandale, R. Losito, E. Bagli, L. Bandiera et al., Phys. Lett. B 719, 70 (2013)

17. A. Mazzolari, E. Bagli, L. Bandiera, V. Guidi et al., Phys. Rev. Lett. 112, 135503 (2014)

18. T.N. Wistisen, U.I. Uggerhøj, U. Wienands, T.W. Markiewicz et al., Phys. Rev. Accel. Beams 19, 071001 (2016)

19. M. Tabrizi, A.V. Korol, A.V. Solov'yov, W. Greiner, Phys. Rev. Lett. 98, 164801 (2007)

20. V.M. Biryukov, (2007). arXiv:0712.3904

21. N.F. Shul'ga, I.V. Kirillin, V.I. Truten', Phys. Lett. B 702, 100 (2011)

22. N.F. Shul'ga, I.V. Kirillin, V.I. Truten', Nuovo Cim. C 34, 425 (2011)

23. I.V. Kirillin, N.F. Shul'ga, L. Bandiera et al., Eur. Phys. J. C. 77, 117 (2017)

24. I.V. Kirillin, Phys. Rev. Accel. Beams 20, 104401 (2017)

25. I.V. Kyryllin, N.F. Shul'ga, Eur. Phys. J. C 79, 1015 (2019)

26. J.F. Bak, A. Burenkov, J.B.B. Petersen et al., Nucl. Phys. B 288, 681 (1987)

27. R.M. Nazhmudinov, A.S. Kubankin, A.V. Shchagin et al., Nucl. Instr. Methods Phys. Res. B 391, 69 (2017)

28. K. Dettmann, M.T. Robinson, Phys. Rev. B 10, 1 (1974)

29. K. Dettmann, Z. Phys. A 272, 227 (1975)

30. H. Esbensen, J.A. Golovchenko, Nucl. Phys. A 298, 382 (1978)

31. E. Fermi, Phys. Rev. 57, 485 (1940) 